AperTO - Archivio Istituzionale Open Access dell'Università di Torino

Weak-Type estimates for the metaplectic representation restricted to the shearing and Dilation subgroup of $S L(2, \mathbb{R})$

This is a pre print version of the following article:

Original Citation:

Availability:

This version is available http://hdl.handle.net/2318/1696166

since 2019-04-03T13:53:45Z

Publisher:

Springer International Publishing

Published version:

DOI:10.1007/978-3-030-05210-2_3

Terms of use:

Open Access

Anyone can freely access the full text of works made available as "Open Access". Works made available under a Creative Commons license can be used according to the terms and conditions of said license. Use of all other works requires consent of the right holder (author or publisher) if not exempted from copyright protection by the applicable law. 


\title{
Weak-type estimates for the metaplectic representation restricted to the shearing and dilation subgroup of $S L(2, \mathbb{R})$
}

\author{
Alessandra Cauli
}

\begin{abstract}
We consider the subgroup $G$ of $S L(2, \mathbb{R})$ consisting of shearing and dilations and we study the decay at infinity of the matrix coefficients of the metaplectic representation restricted to $G$. We prove weak-type estimates for such coefficients, which are uniform for functions in the modulation space $M^{1}$. This work represents a continuation of a project aiming at studying weak-type and Strichartz estimates for unitary representations of non-compact Lie groups.
\end{abstract}

\section{Introduction}

In [1] we started a project aiming at studying weak-type and Strichartz estimates for unitary representations of non-compact Lie groups. There, we consider the case of the metaplectic representation, which is a faithful representation of the metaplectic group $M p(n, \mathbb{R})$ in $L^{2}\left(\mathbb{R}^{n}\right)$, being the double covering of the symplectic group $S p(n, \mathbb{R})$. It can therefore be identified with a subgroup of unitary operators $\hat{S}$ on $L^{2}\left(\mathbb{R}^{n}\right)$. We denote by $\hat{S} \mapsto S \in S p(n, \mathbb{R})$ the projection. Among other things, we proved the uniform and sharp weak-type estimate

$$
\left\|\left\langle\hat{S} \varphi_{1}, \varphi_{2}\right\rangle\right\|_{L^{4, \infty}(M p(n, \mathbb{R}))} \lesssim\left\|\varphi_{1}\right\|_{M^{1}}\left\|\varphi_{2}\right\|_{M^{1}}
$$

where $M p(n, \mathbb{R})$ is endowed with its Haar measure. In this formula $M^{1}$ denotes a modulation space - a Banach space well-known in Time-frequency Analysis [16] - whose definition will be recalled in the next section. Here it is enough to observe that it is dense in $L^{2}\left(\mathbb{R}^{n}\right)$ and contains the Schwartz space. From the above estimate,

Alessandra Cauli

Dipartimento di Scienze Matematiche, Politecnico di Torino

e-mail: alessandra.cauli@polito.it 
we can deduce Strichartz-type estimates for the metaplectic representation. In this paper we consider a similar problem, but for the metapletic representation restricted to the shearing and dilation subgroup $G$ of $S L(2, \mathbb{R})$, constituted by the matrices of the form $l_{t} d_{s^{1 / 2}}$, with $t \in \mathbb{R}, s>0$, where

$$
d_{s}=\left(\begin{array}{cc}
s^{-1} & 0 \\
0 & s
\end{array}\right), s>0, l_{t}=\left(\begin{array}{ll}
1 & 0 \\
t & 1
\end{array}\right), t \in \mathbb{R}
$$

hence

$$
l_{t} d_{s^{1 / 2}}=\left(\begin{array}{ll}
1 & 0 \\
t & 1
\end{array}\right)\left(\begin{array}{cc}
s^{-1 / 2} & 0 \\
0 & s^{1 / 2}
\end{array}\right)=\left(\begin{array}{cc}
s^{-1 / 2} & 0 \\
t s^{-1 / 2} & s^{1 / 2}
\end{array}\right)
$$

The corresponding Haar measure is $\frac{d t d s}{s^{2}}$. This group is particularly important in Time-frequency Analysis because it is reproducing, see [13, Lemma 2.1] as well as [2],[3],[4] and [5] where it is shown that the metaplectic representation plays a key role in the classification of the reproducing subgroups of the affine symplectic group.

Now, for any $\varphi_{1}, \varphi_{2} \in M^{1}$ we consider again the matrix coefficient $G \rightarrow \mathbb{R}, S \mapsto$ $\left|\left\langle\hat{S} \varphi_{1}, \varphi_{2}\right\rangle\right|$. Our main result reads as follows.

Theorem 1. Given $G$ as above, we have:

$$
\left\|\left\langle\hat{S} \varphi_{1}, \varphi_{2}\right\rangle\right\|_{L^{4, \infty}} \lesssim\left\|\varphi_{1}\right\|_{M^{1}}\left\|\varphi_{2}\right\|_{M^{1}}
$$

From this result it is easy to obtain corresponding Strichartz-type estimates, following the pattern in [1]. The results on Strichartz estimates are often applied to wellposedness and scattering of nonlinear PDEs, see [17] for further details. We also refer to [10], [11] and [12] for general results concerning decay estimates for matrix coefficients of unitary representation and to $[6,7,8,9]$ for the role of the metaplectic representation in PDEs from a Time-frequency Analysis perspective.

The paper is organized as follows. Section 2 contains some preliminary results, Section 3 is devoted to a survey of the results on reproducing groups, with the aim of describe the role of the above group $G$ in Time-frequency Analysis. In section 4 we recall some results from [1], while in Section 5 we prove our main result.

\section{Preliminaries}

\subsection{Integration on the symplectic group}

The symplectic group $S p(n, \mathbb{R})$ is the group of $2 n \times 2 n$ real matrices $S$ such that $S J=J S$, where 


$$
J=\left(\begin{array}{cc}
0 & I_{n} \\
-I_{n} & 0
\end{array}\right)
$$

is the standard symplectic form

$$
\omega(x, y)=x^{T} J y, \quad x, y \in \mathbb{R}^{2 n} .
$$

$S p(n, \mathbb{R})$ turns out to be a unimodular Lie group. The following integration formula for $U(2 n, \mathbb{R})$-bi-invariant functions on $S p(n, \mathbb{R})$ will be crucial in the following.

Recall that $f: S p(n, \mathbb{R}) \rightarrow \mathbb{C}$ is called $U(2 n, \mathbb{R})$-bi-invariant if $f\left(U_{1} S U_{2}\right)=f(S)$ for every $S \in S p(n, \mathbb{R}), U_{1}, U_{2} \in U(2 n, \mathbb{R})$.

Consider the Abelian subgroup $A=\left\{a_{t}\right\}$ of $S p(n, \mathbb{R})$ given by

$$
a_{t}=\left(\begin{array}{cc}
e^{\frac{t}{2}} & 0 \\
0 & e^{-\frac{t}{2}}
\end{array}\right), \quad t=\operatorname{diag}\left(t_{1}, \ldots, t_{n}\right),\left(t_{1}, \ldots, t_{n}\right) \in \mathbb{R}^{n} .
$$

If $f$ is a $U(2 n, \mathbb{R})$-bi-invariant function on $\operatorname{Sp}(n, \mathbb{R})$, its integral with respect to the Haar measure is given by

$$
\begin{aligned}
\int_{S p(n, \mathbb{R})} f(S) d S & \\
& =C \int_{t_{1} \geq \ldots \geq t_{n} \geq 0} f\left(a_{t}\right) \prod_{i<j} \sinh \frac{t_{i}-t_{j}}{2} \prod_{i \leq j} \sinh \frac{t_{i}+t_{j}}{2} d t_{1} \ldots d t_{n}
\end{aligned}
$$

for some constant $C>0$.

\subsection{The metaplectic representation}

The metaplectic representation $\mu$ links the standard Schrödinger representation $\rho$ of the Heisenberg group $\mathbb{H}^{n}$ to the representation obtained from it by composing $\rho$ with the action of $\operatorname{Sp}(n, \mathbb{R})$ by automorphisms on $\mathbb{H}^{n}$. We recall here its construction. The product in the Heisenberg group $\mathbb{H}^{n}$ is defined by

$$
(z, t) \cdot\left(z^{\prime}, t^{\prime}\right)=\left(z+z^{\prime}, t+t^{\prime}-\frac{1}{2} \omega\left(z, z^{\prime}\right)\right)
$$

on $\mathbb{R}^{2 n+1}$, where $\omega$ is the standard symplectic form in $\mathbb{R}^{2 n}$ given by (1). We denote the translation and modulation operators on $L^{2}\left(\mathbb{R}^{n}\right)$ by

$$
T_{x} f(t)=f(t-x) \quad \text { and } \quad M_{\xi} f(t)=e^{2 \pi i\langle\xi, t\rangle} f(t) .
$$

The Schrödinger representation of the group $\mathbb{H}^{n}$ on $L^{2}\left(\mathbb{R}^{n}\right)$ is then defined by

$$
\rho(x, \xi, t) f(y)=e^{2 \pi i t} e^{\pi i\langle x, \xi\rangle} e^{2 \pi i\langle\xi, y-x\rangle} f(y-x)=e^{2 \pi i t} e^{\pi i\langle x, \xi\rangle} T_{x} M_{\xi} f(y),
$$


where we write $z=(x, \xi)$ when we separate space components from frequency components in a point $z$ in the phase space $\mathbb{R}^{2 n}$. The symplectic group acts on $\mathbb{H}^{n}$ via automorphisms that leave the center $\{(0, t): t \in \mathbb{R}\} \simeq \mathbb{R}$ of $\mathbb{H}^{n}$ point-wise fixed:

$$
A \cdot(z, t)=(A z, t) .
$$

Moreover, for any fixed $A \in \operatorname{Sp}(n, \mathbb{R})$ there is a representation

$$
\rho_{A}: \mathbb{H}^{n} \longrightarrow \mathscr{U}\left(L^{2}\left(\mathbb{R}^{n}\right)\right), \quad(z, t) \mapsto \rho(A \cdot(z, t))
$$

whose restriction to the center is a multiple of the identity. By the Stone-von Neumann theorem, $\rho_{A}$ is equivalent to $\rho$. This means that there exists an unitary operator $\mu(A) \in \mathscr{U}\left(L^{2}\left(\mathbb{R}^{n}\right)\right)$ such that $\rho_{A}(z, t)=\mu(A) \circ \rho(z, t) \circ \mu(A)^{-1}$, for all $(z, t) \in \mathbb{H}^{n}$. By Schur's lemma, $\mu$ is determined up to a phase factor $e^{i s}, s \in \mathbb{R}$. Actually, the phase ambiguity is really a sign and $\mu$ lifts to a representation of the double cover of the symplectic group. It is exactly the famous metaplectic or Shale-Weil representation.

The representations $\rho$ and $\mu$ can be combined and produce the extended metaplectic representation of the group $G=\mathbb{H}^{n} \rtimes S p(n, \mathbb{R})$. The group law on $G$ is

$$
((z, t), A) \cdot\left(\left(z^{\prime}, t^{\prime}\right), A^{\prime}\right)=\left((z, t) \cdot\left(A z^{\prime}, t^{\prime}\right), A A^{\prime}\right)
$$

and the extended metaplectic representation $\mu_{e}$ of $G$ is

$$
\mu_{e}((z, t), A)=\rho(z, t) \circ \mu(A) .
$$

The role of the center of the Heisenberg group is irrelevant and the true group under consideration is $\mathbb{R}^{2 n} \rtimes S p(n, \mathbb{R})$, which we denote again by $G$. $G$ acts naturally by affine transformations on the phase space, namely

$$
g \cdot(x, \xi)=((q, p), A) \cdot(x, \xi)=A^{T}(x, \xi)+(q, p)^{T} .
$$

For elements of $\operatorname{Sp}(n, \mathbb{R})$ in special form and for $f \in L^{2}\left(\mathbb{R}^{n}\right)$, the metaplectic representation can be computed explicitly in a simple way, so we have:

$$
\begin{gathered}
\mu\left(\left[\begin{array}{cc}
A & 0 \\
0 & \left(A^{T}\right)^{-1}
\end{array}\right]\right) f(x)=(\operatorname{det} A)^{-1 / 2} f\left(A^{-1} x\right) \\
\mu\left(\left[\begin{array}{ll}
I & 0 \\
C & I
\end{array}\right]\right) f(x)= \pm e^{i \pi\langle C x, x\rangle} f(x) \\
\mu(J)=(-i)^{d / 2} \mathscr{F}
\end{gathered}
$$

where $\mathscr{F}$ denotes the Fourier transform

$$
\mathscr{F} f(\xi)=\int_{\mathbb{R}^{n}} f(x) e^{-2 \pi i\langle x, \xi\rangle} d x, \quad f \in L^{1}\left(\mathbb{R}^{n}\right) \cap L^{2}\left(\mathbb{R}^{n}\right) .
$$




\subsection{Modulation spaces}

Fix a window function $\varphi \in \mathscr{S}\left(\mathbb{R}^{n}\right) \backslash\{0\}$. The short-time Fourier transform (STFT) of a function/temperate distribution $\psi \in \mathscr{S}^{\prime}\left(\mathbb{R}^{n}\right)$ with respect to $\varphi$ is defined by

$$
V_{\varphi} \psi(x, \xi)=\int_{\mathbb{R}^{n}} e^{-2 \pi i \xi \cdot y} \psi(y) \overline{\varphi(y-x)} d y, \quad x, \xi \in \mathbb{R}^{n}
$$

For $1 \leq p, q \leq \infty$ and a Schwartz function $\varphi \in \mathscr{S}\left(\mathbb{R}^{n}\right) \backslash\{0\}$, the modulation space $M^{p, q}\left(\mathbb{R}^{n}\right)$ is defined as the space of $\psi \in \mathscr{S}^{\prime}\left(\mathbb{R}^{n}\right)$ such that

$$
\|\psi\|_{M^{p, q}}:=\left(\int_{\mathbb{R}^{n}}\left(\int_{\mathbb{R}^{n}}\left|V_{\varphi} \psi(x, \xi)\right|^{p} d x\right)^{q / p} d \xi\right)^{1 / q}<\infty
$$

with obvious changes if $p=\infty$ or $q=\infty$.

If $p=q$, then we write $M^{p}$ instead of $M^{p, p}$.

We will also need a variant, sometimes called Wiener amalgam space in the literature, whose norm is

$$
\|\psi\|_{W\left(F L^{p}, L^{q}\right)}:=\left(\int_{\mathbb{R}^{n}}\left(\int_{\mathbb{R}^{n}}\left|V_{\varphi} \psi(x, \xi)\right|^{p} d \xi\right)^{q / p} d x\right)^{1 / q}
$$

here the Lebesgue norms appear in the inverse order. Both these norms provide a measure of the time-frequency concentration of a function and are widely used in Time-frequency Analysis [14, 16].

We have $M^{p_{1}, q_{1}} \subseteq M^{p_{2}, q_{2}}$ if and only if $p_{1} \leq p_{2}$ and $q_{1} \leq q_{2}$. Similarly, $W\left(F L^{p_{1}}, L^{q_{1}}\right) \subseteq$ $W\left(F L^{p_{2}}, L^{q_{2}}\right)$ if and only if $p_{1} \leq p_{2}$ and $q_{1} \leq q_{2}$.

The duality goes as expected:

$$
\left(M^{p, q}\right)^{\prime}=M^{p^{\prime}, q^{\prime}}, \quad 1 \leq p, q<\infty
$$

and in particular

$$
|\langle f, g\rangle| \lesssim\|f\|_{M^{p}}\|g\|_{M^{p^{\prime}}}
$$

In the dispersive estimates we meet, in particular, the Gelfand triple

$$
M^{1} \subset L^{2}\left(\mathbb{R}^{n}\right) \subset M^{\infty} .
$$

We observe that

$$
\mathscr{S}\left(\mathbb{R}^{n}\right) \subset M^{1} \subset L^{2}\left(\mathbb{R}^{n}\right)
$$

with dense and strict inclusions. For atomic characterizations of the space $M^{1}$ we refer to $[14,16]$.

We will also use the complex interpolation theory for modulation spaces, which reads as follows: for $1 \leq p, q, p_{i}, q_{i} \leq \infty, i=0,1,0 \leq \theta \leq 1$,

$$
\frac{1}{p}=\frac{1-\vartheta}{p_{0}}+\frac{\vartheta}{p_{1}}, \quad \frac{1}{q}=\frac{1-\vartheta}{q_{0}}+\frac{\vartheta}{q_{1}},
$$


we have

$$
\left(M^{p_{0}, q_{0}}, M^{p_{1}, q_{1}}\right)_{\vartheta}=M^{p, q} .
$$

\section{The role of the metaplectic representation in Time-frequency Analysis}

The metaplectic representation became an essential tool in Time-frequency Analysis as it is very important in Weyl pseudo-differential calculus, and it is strictly related to a quadratic time-frequency representation called Wigner distribution, which is regarded as one of the best mathematical description of the time-frequency behavior of signals. The metaplectic representation is also important in order to understand when a subgroup is reproducing as we will see in the following.

\subsection{The Wigner distribution and some of its properties}

In this paragraph, we recall some basic definition and properties; we refer to [14] and [15] for more details.

Definition 1. The Wigner distribution $W(\varphi)$ of a function $\varphi \in L^{2}\left(\mathbb{R}^{n}\right)$ is defined to be

$$
W \varphi(x, \xi)=\int_{\mathbb{R}^{n}} \varphi\left(x+\frac{t}{2}\right) \overline{\varphi\left(x-\frac{t}{2}\right)} e^{-2 \pi i \xi \cdot t} d t .
$$

By polarizing the quadratic expression, one obtains the cross-Wigner distribution of $\varphi, \psi \in L^{2}\left(\mathbb{R}^{n}\right)$ :

$$
W(\varphi, \psi)(x, \xi)=\int_{\mathbb{R}^{n}} \varphi\left(x+\frac{t}{2}\right) \overline{\psi\left(x-\frac{t}{2}\right)} e^{-2 \pi i \xi \cdot t} d t
$$

We also set $W(\varphi)=W(\varphi, \varphi)$.

Proposition 1. The cross-Wigner distribution of $\varphi, \psi \in L^{2}\left(\mathbb{R}^{n}\right)$ satisfies:

1. $W(\varphi, \psi)$ is uniformly continuous on $\mathbb{R}^{2 n}$ and $\|W(\varphi, \psi)\|_{\infty} \leq 2^{n}\|\varphi\|_{2}\|\psi\|_{2}$.

2. $W(\varphi, \psi)=\overline{W(\psi, \varphi)}$; in particular, $W(\varphi)$ is real-valued.

3. For $u, v, \eta, \gamma \in \mathbb{R}^{n}$, we have

$$
\begin{gathered}
W\left(T_{u} M_{\eta} \varphi, T_{v} M_{\gamma} \psi\right)(x, \xi)= \\
=e^{\pi i(u+v) \cdot(\gamma-\eta)} e^{2 \pi i x \cdot(\eta-\gamma)} e^{-2 \pi i \xi \cdot(u-v)} \cdot W(\varphi, \psi)\left(x-\frac{u+v}{2}, \xi-\frac{\eta+\gamma}{2}\right) .
\end{gathered}
$$

In particular, $W \varphi$ is covariant, that is,

$$
W\left(T_{u} M_{\eta} \varphi\right)(x, \xi)=W \varphi(x-u, \xi-\eta)
$$


4. $W(\hat{\varphi}, \hat{\psi})(x, \xi)=W(\varphi, \psi)(-\xi, x)$.

5. Moyal's formula: for $\varphi_{1}, \varphi_{2}, \psi_{1}, \psi_{2} \in L^{2}\left(\mathbb{R}^{n}\right)$,

$$
\left\langle W\left(\varphi_{1}, \psi_{1}\right), W\left(\varphi_{2}, \psi_{2}\right)\right\rangle_{L^{2}\left(\mathbb{R}^{2 n}\right)}=\left\langle\varphi_{1}, \varphi_{2}\right\rangle \overline{\left\langle\psi_{1}, \psi_{2}\right\rangle} .
$$

6. Marginal densities: If $\varphi, \hat{\varphi} \in L^{1} \cap L^{2}\left(\mathbb{R}^{n}\right)$, then

$$
\int_{\mathbb{R}^{n}} W \varphi(x, \xi) d \xi=|\varphi(x)|^{2}, \int_{\mathbb{R}^{n}} W \hat{\varphi}(x, \xi) d \xi=|\hat{\varphi}(x)|^{2} .
$$

In particular,

$$
\iint_{\mathbb{R}^{2 n}} W \varphi(x, \xi) d x d \xi=\|\varphi\|_{2}^{2}
$$

\subsection{Reproducing groups}

We now come to the notion of reproducing groups and we show how the metaplectic representation is essential in their characterization. We consider a Lie group $H$ with left Haar measure $d h$ and $\phi \in L^{2}\left(\mathbb{R}^{n}\right)$ is a unitary representation of $H$ in $L^{2}\left(\mathbb{R}^{n}\right)$, and we are interested in reproducing formulae of the type:

$$
f=\int_{H}\left\langle f, \phi_{h}\right\rangle \phi_{h} d h, \quad f \in \mathscr{H},
$$

In particular, we consider the case when the Lie group $H$ in (10) is a subgroup of the semi-direct product $G=\mathbb{H}^{n} \rtimes S p(n, \mathbb{R})$ of the Heisenberg group and the symplectic group, while the representation $h \mapsto \phi_{h}$ arises from the restriction to $H$ of the reducible (extended) metaplectic representation $\mu_{e}$ of $G$ as applied to a fixed and suitable window function $\phi \in L^{2}\left(\mathbb{R}^{n}\right)$. A group $H$ for which there exists a window $\phi$ such that (10) holds is said to be reproducing.

Definition 2. We say that a connected Lie subgroup $H$ of $G=\mathbb{R}^{2 n} \rtimes S p(n, \mathbb{R})$ is a reproducing group for $\mu_{e}$ if there exists a function $\phi \in L^{2}\left(\mathbb{R}^{n}\right)$ such that

$$
f=\int_{H}\left\langle f, \mu_{e}(h) \phi\right\rangle \mu_{e}(h) \phi d h, \quad \text { for } \text { all } f \in L^{2}\left(\mathbb{R}^{n}\right) .
$$

Any $\phi \in L^{2}\left(\mathbb{R}^{n}\right)$ for which (11) holds is called a reproducing function.

Remark 1. We notice that we do require formula (11) to hold for all functions in $L^{2}\left(\mathbb{R}^{n}\right)$ for the same window $\phi$, but we do not require the restriction of $\mu_{e}$ to $H$ to be irreducible.

One of the most important features of $\mu_{e}$ is that it may be realized by affine actions on $\mathbb{R}^{2 n}$ by means of the Wigner distribution. Since the reproducing formula is insensitive to phase factors, i.e., to the action of the center of $\mathbb{H}^{n}$, the group $G$ is truly $\mathbb{R}^{2 n} \rtimes S p(n, \mathbb{R})$. The following notion of admissible subgroup $H$ of $G=$ 
$\mathbb{H}^{n} \rtimes S p(n, \mathbb{R})$ relative to the extended metaplectic representation $\mu_{e}$ via the Wigner distribution is important to establish when a subgroup is reproducing, together with some additional integrability and boundedness properties of $W(\psi)\left(h^{-1} \cdot(x, \xi)\right)$.

Definition 3. We say that a connected Lie subgroup $H$ of $G=\mathbb{R}^{2 n} \rtimes S p(n, \mathbb{R})$ is an admissible group for $\mu_{e}$ if there exists a function $\phi \in L^{2} \mathbb{R}^{n}$ such that

$$
\int_{H} W(\phi)\left(h^{-1} \cdot(x, \xi)\right) d h=1 \quad \text { for } \quad \text { a.e. } \quad(x, \xi) \in \mathbb{R}^{2 n} .
$$

Any $\phi \in L^{2}\left(\mathbb{R}^{n}\right)$ for which (12) holds is called an admissible function.

Theorem 2. Suppose that $\phi \in L^{2}\left(\mathbb{R}^{n}\right)$ is such that the mapping

$$
h \mapsto W\left(\mu_{e}(h) \phi\right)(x, \xi)=W(\phi)\left(h^{-1} \cdot(x, \xi)\right)
$$

is in $L^{1}(H)$ for a.e. $(x, \xi) \in \mathbb{R}^{2 n}$ and

$$
\int_{H}\left|W(\phi)\left(h^{-1} \cdot(x, \xi)\right)\right| d h \leq M, \quad \text { for } \text { a.e. } \quad(x, \xi) \in \mathbb{R}^{2 n} .
$$

The condition (11) holds for all $f \in L^{2}\left(\mathbb{R}^{n}\right)$ if and only if the following admissibility condition is satisfied:

$$
\int_{H} W(\phi)\left(h^{-1} \cdot(x, \xi)\right) d h=1 \quad \text { for } \quad \text { a.e. } \quad(x, \xi) \in \mathbb{R}^{2 n} .
$$

We now dispose of two different tools for checking whether a subgroup $H$ of $G=\mathbb{R}^{2 n} \rtimes S p(n, \mathbb{R})$ is reproducing or not. Either we find a window function $\phi$ for which (11) holds or we check the admissibility of the subgroup $H$ and use Theorem 2 .

\section{On dispersive and Strichartz estimates for the metaplectic representation}

We recall here a number of definitions and results from [1] that we will use in the following. As anticipated in the introduction, in [1] we proved the following dispersive-type estimate.

Theorem 3 (Dispersive estimate). The following estimate holds:

$$
\|\widehat{S} \psi\|_{M^{\infty}} \lesssim\left(\lambda_{1}(S) \ldots \lambda_{n}(S)\right)^{-1 / 2}\|\psi\|_{M^{1}}
$$

for $\widehat{S} \in M p(n, \mathbb{R}), \psi \in \mathscr{S}\left(\mathbb{R}^{n}\right)$, where $\lambda_{1}(S), \ldots, \lambda_{n}(S)$ are the singular values $\geq 1$ of $S=\pi(\widehat{S}) \in S p(n, \mathbb{R})$.

By duality this is in fact equivalent to: 


$$
|\langle\widehat{S} \psi, \varphi\rangle| \lesssim\left(\lambda_{1}(S) \ldots \lambda_{n}(S)\right)^{-1 / 2}\|\psi\|_{M^{1}}\|\varphi\|_{M^{1}}, \forall \widehat{S} \in M p(n, \mathbb{R})
$$

Corollary 1 (Uniform weak-type estimate for matrix coefficients). Let $G=$ $\operatorname{Mp}(n, \mathbb{R})$ with the Haar measure. The following estimate holds:

$$
\left\|\left\langle\widehat{S} \varphi_{1}, \varphi_{2}\right\rangle\right\|_{L^{4 n, \infty}(G)} \lesssim\left\|\varphi_{1}\right\|_{M^{1}}\left\|\varphi_{2}\right\|_{M^{1}}
$$

for $\varphi_{1}, \varphi_{2} \in \mathscr{S}\left(\mathbb{R}^{n}\right)$.

The proof of the above corollary is in fact based on theorem 3 and the following result:

Proposition 2. Let $\alpha>0, \beta>0$. Consider the function

$$
h(S)=\left(\lambda_{1}(S) \ldots \lambda_{n}(S)\right)^{-\alpha}
$$

on $\operatorname{Sp}(n, \mathbb{R})$, where $\lambda_{1}(S) \ldots \lambda_{n}(S)$ are the singular values $\geq 1$ of the symplectic matrix $S$.

We have $h \in L^{\beta, \infty}$ on $\operatorname{Sp}(n, \mathbb{R})$, with respect to the Haar measure, if

$$
\alpha \beta \geq 2 n \text {. }
$$

Proposition 2 is in turn proved by using the explicit formula for the integration of functions on $M p(n, \mathbb{R})$ (i.e. $S p(n, \mathbb{R})$ ), that we recalled in the previous Section.

Finally, as a consequence of the dispersive estimates we therefore obtained the following Strichartz-type estimates.

Theorem 4 (Strichartz estimates). Let $G=M p(n, \mathbb{R})$ with the Haar measure. The following estimates hold:

$$
\|\widehat{S} \psi\|_{L^{q}\left(G ; M^{r}\right)} \lesssim\|\psi\|_{L^{2}}
$$

for

$$
\frac{4 n}{q}+\frac{1}{r} \leq \frac{1}{2}, \quad 2 \leq q, r \leq \infty .
$$

The range of admissible pairs $(q, r)$ in Theorem 4 is represented in Figure 4.

For the sake of completeness, we now report a sketch of the proof of the previous theorem.

Proof (Proof of Theorem 4). We know that

$$
\|\widehat{S} \psi\|_{L^{2}}=\|\psi\|_{L^{2}}
$$

for $\psi \in L^{2}\left(\mathbb{R}^{n}\right)$, which gives the desired Strichartz estimate for $q=\infty, r=2$, because $M^{2}=L^{2}$, and also for $q=\infty, 2 \leq r \leq \infty$, because $L^{2} \hookrightarrow M^{r}$ for $r \geq 2$. Hence from now on we can suppose $q<\infty$.

Now by Theorem 3 , 


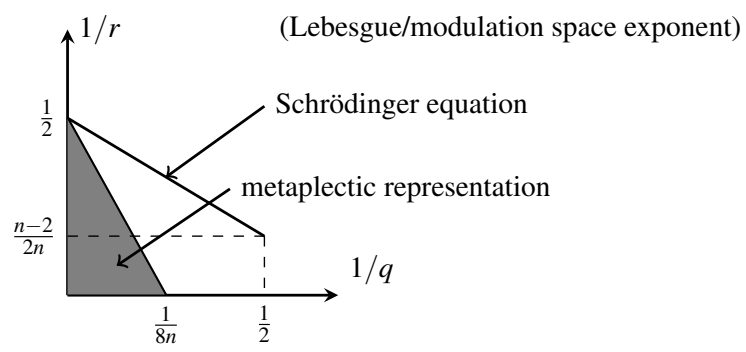

Fig. 1 Admissible pairs for Strichartz estimates

$$
\|\widehat{S} \psi\|_{M^{\infty}} \lesssim\left(\lambda_{1}(S) \ldots \lambda_{n}(S)\right)^{-1 / 2}\|\psi\|_{M^{1}} .
$$

By interpolation with (19) we obtain, for every $2 \leq r \leq \infty$,

$$
\|\widehat{S} \psi\|_{M^{r}} \lesssim\left(\lambda_{1}(S) \ldots \lambda_{n}(S)\right)^{-\left(\frac{1}{2}-\frac{1}{r}\right)}\|\psi\|_{M^{\prime}} .
$$

Let now $G=M p(n, \mathbb{R})$, as in the statement. Then one concludes by applying the usual $T T^{*}$ method (see [17, page 75]) to the operator

$$
T: L^{2} \rightarrow L^{q}\left(G ; M^{r}\right), T \psi=\widehat{S} \psi .
$$

\section{Proof of the main results}

Since $G \subset S L(2, \mathbb{R})=S p(1, \mathbb{R})$, the estimate (17) holds with $n=1$ for $S \in G$. Let now

$$
S=l_{t} d_{s^{1 / 2}}=\left(\begin{array}{cc}
s^{-1 / 2} & 0 \\
t s^{-1 / 2} & s^{1 / 2}
\end{array}\right)
$$

be a generic element of $G$, so that

$$
S^{*}=\left(\begin{array}{cc}
s^{-1 / 2} & t s^{-1 / 2} \\
0 & s^{1 / 2}
\end{array}\right)
$$

and

$$
S^{*} S=\left(\begin{array}{cc}
s^{-1 / 2} & t s^{-1 / 2} \\
0 & s^{1 / 2}
\end{array}\right)\left(\begin{array}{cc}
s^{-1 / 2} & 0 \\
t s^{-1 / 2} & s^{1 / 2}
\end{array}\right)=\left(\begin{array}{cc}
s^{-1}+t^{2} s^{-1} & t \\
t & s
\end{array}\right) .
$$

We determine the singular values of $S$, which are the square roots of the eigenvalues of $S^{*} S$. Now,

$$
\operatorname{det}\left(S^{*} S-\lambda I\right)=\lambda^{2}-\left(s+s^{-1}+t^{2} s^{-1}\right) \lambda+1
$$

from which 


$$
\lambda_{1,2}=\frac{s+s^{-1}+t^{2} s^{-1} \pm \sqrt{\left(s-s^{-1}\right)^{2}+t^{2}\left(t^{2} s^{-2}+2 s^{-2}+2\right)}}{2} .
$$

We assume $\lambda_{2} \leq \lambda_{1}$ and we have $\lambda_{2}=\lambda_{1}^{-1}$, so that $\lambda_{1} \geq 1$. We get:

$$
\lambda_{1}^{-1 / 2}=\left(\frac{1}{2}\right)^{-1 / 2}\left(s+s^{-1}\left(1+t^{2}\right)+\sqrt{\left(s-s^{-1}\right)^{2}+t^{2} s^{-2}\left(t^{2}+2\right)+2 t^{2}}\right)^{-1 / 2} .
$$

Let us observe that

$$
\sqrt{\left(s-s^{-1}\right)^{2}+t^{2} s^{-2}\left(t^{2}+2\right)+2 t^{2}} \asymp\left|s-s^{-1}\right|+|t| s^{-1} \sqrt{t^{2}+1}+|t| .
$$

We have to estimate the measure of the set

$$
D_{\lambda}=\left\{(t, s) \in \mathbb{R} \times \mathbb{R}_{+}: \lambda_{1}(t, s)^{-1 / 4} \geq \lambda\right\},
$$

$\lambda>0$ with respect to the Haar measure $\frac{d t d s}{s^{2}}$ i.e.

$$
\int_{\mathbb{R} \times \mathbb{R}_{+}} \chi_{D_{\lambda}} \frac{d t d s}{s^{2}}
$$

where $\chi_{D_{\lambda}}$ is the indicator function of $D_{\lambda}$. We split the domain of the indicator function into several regions. As $D_{\lambda}=\emptyset$ if $\lambda>1$, we will consider $\lambda \leq 1$ in the following.

Let us consider $D_{1}:|t| \leq 2, s \geq 2$. Then we have:

$$
s+s^{-1}\left(1+t^{2}\right) \asymp s+s^{-1} \asymp s
$$

and

$$
\begin{aligned}
\left|s-s^{-1}\right|+|t| s^{-1} \sqrt{t^{2}+1}+|t| & \asymp s+|t| s^{-1}+|t|=s+|t|\left(s^{-1}+1\right) \\
& \asymp s+|t| \asymp s .
\end{aligned}
$$

Hence,

$$
\lambda_{1}(t, s) \asymp s .
$$

Since

$$
s^{-1 / 4} \geq \lambda \Leftrightarrow s \leq \lambda^{-4},
$$

we have 


$$
\int_{D_{1}} \chi_{D_{1}} \frac{d t d s}{s^{2}} \leq \int_{|t| \leq 2,2 \leq s \lesssim \lambda-4} \frac{d t d s}{s^{2}}=\mathscr{O}(1)
$$

as $\lambda \rightarrow 0^{+}$.

Then consider $D_{2}:|t| \geq 2, s>2$. We have:

$$
s+s^{-1}\left(1+t^{2}\right) \asymp s+s^{-1} t^{2}
$$

and

$$
\left|s-s^{-1}\right|+|t| s^{-1} \sqrt{t^{2}+1}+|t| \asymp s+|t|\left(1+|t| s^{-1}\right) .
$$

Hence

$$
\lambda_{1}(t, s) \asymp s+|t|\left(1+s^{-1}|t|\right) .
$$

We consider two cases:

- If $s^{-1}|t| \geq 1, \lambda_{1}(t, s) \asymp s+s^{-1} t^{2}$.

Clearly,

$$
s+s^{-1} t^{2} \geq s^{-1} t^{2}
$$

and

$$
\left(s^{-1} t^{2}\right) \geq \lambda^{2}
$$

if

$$
|t| \leq \lambda^{-1} s^{-1 / 2}
$$

Then,

$$
\begin{aligned}
\int_{D_{2}} \chi_{D_{2}} \frac{d t d s}{s^{2}} & \leq \int_{2}^{+\infty} \frac{1}{s^{2}} \int_{|t| \lesssim \lambda-1 s^{-1 / 2}} d t d s \\
& \asymp \lambda^{-1} \int_{2}^{+\infty} \frac{1}{s^{3 / 2}} d s=\mathscr{O}\left(\lambda^{-1}\right) .
\end{aligned}
$$

- If $s^{-1}|t| \leq 1, \lambda_{1}(t, s) \asymp s+|t|$.

Since

$$
\begin{aligned}
s+|t| & \leq \lambda^{-4} \Rightarrow|t| \leq \lambda^{-4} \\
\int_{D_{2}} \chi_{D_{2}} \frac{d t d s}{s^{2}} & \leq \int_{|t| \lesssim \lambda^{-4}, s \geq 2} \frac{d t d s}{s^{2}} \\
& \lesssim \lambda^{-4} \int_{2}^{+\infty} \frac{d s}{s^{2}}=\mathscr{O}\left(\lambda^{-4}\right)
\end{aligned}
$$

So we have

$$
\int_{D_{2}} \chi_{D_{2}} \frac{d t d s}{s^{2}} \lesssim \lambda^{-4}
$$

Then consider $D_{3}:|t| \geq 2,0<s<\frac{1}{2}$. Then we have: 
Weak-type estimates

$$
s+s^{-1}\left(1+t^{2}\right) \asymp s+s^{-1} t^{2} \asymp s+s^{-1} t^{2}
$$

and

$$
\begin{aligned}
\left|s-s^{-1}\right|+|t| s^{-1} \sqrt{t^{2}+1}+|t| & \asymp s^{-1}+t^{2} s^{-1}+|t|=s^{-1}\left(1+t^{2}\right)+|t| \\
& \asymp s^{-1} t^{2}+|t|=|t|\left(s^{-1}|t|+1\right) \asymp s^{-1} t^{2} .
\end{aligned}
$$

So,

$$
\lambda_{1}(t, s) \asymp s+s^{-1} t^{2}
$$

and

$$
s+s^{-1} t^{2} \leq \lambda^{-4}
$$

implies

$$
|t| \leq s^{1 / 2} \lambda^{-2}
$$

Then,

$$
\int_{D_{3}} \chi_{D_{3}} \frac{d t d s}{s^{2}} \leq \int_{\lambda^{2} \lesssim s \leq 1 / 2} \frac{1}{s^{2}} \int_{2 \leq|t| \leqslant s^{1 / 2} \lambda^{-2}} d t d s \asymp \lambda^{-2} .
$$

Consider $D_{4}:|t| \geq 2, \frac{1}{2}<s<2$. So we have:

$$
s+s^{-1}\left(1+t^{2}\right) \asymp t^{2}
$$

and

$$
\left|s-s^{-1}\right|+|t| s^{-1} \sqrt{t^{2}+1}+|t| \asymp|s-1|+t^{2} \asymp t^{2} .
$$

On the other hand,

$$
t^{2} \leq \lambda^{-4} \Rightarrow|t| \leq \lambda^{-2}
$$

and therefore

$$
\int_{D_{4}} \chi_{D_{4}} \frac{d t d s}{s^{2}} \lesssim \int_{2 \leq|t| \lesssim \lambda^{-2}, \frac{1}{2}<s<2} 1 \frac{d t d s}{s^{2}} \asymp \lambda^{-2} .
$$

Consider $D_{5}:|t| \leq 2,0<s<\frac{1}{2}$. Then we have:

$$
s+s^{-1}\left(1+t^{2}\right) \asymp s+s^{-1} \asymp s^{-1}
$$

and

$$
\begin{aligned}
\left|s-s^{-1}\right|+|t| s^{-1} \sqrt{t^{2}+1}+|t| & \asymp\left|s-s^{-1}\right|+|t| s^{-1} \sqrt{t^{2}+1}+|t| \\
& \asymp s^{-1}+|t| s^{-1}+|t|=s^{-1}(|t|+1)+|t| \asymp s^{-1}+|t| .
\end{aligned}
$$

Hence

$$
\lambda_{1}(t, s) \asymp s^{-1}+|t|
$$

and

$$
s^{-1}+|t| \leq \lambda^{-4} \Rightarrow s^{-1} \leq \lambda^{-4} \Rightarrow s \geq \lambda^{4}
$$


so that

$$
\int_{D_{5}} \chi_{D_{5}} \frac{d t d s}{s^{2}} \leq \int_{|t| \leq 2, \lambda^{4} \lesssim s<\frac{1}{2}} \frac{d t d s}{s^{2}} \lesssim \lambda^{-4} .
$$

Consider $D_{6}:|t| \leq 2, \frac{1}{2}<s<2$. Then we have:

$$
\int_{D_{6}} \chi_{D_{6}} \frac{d t d s}{s^{2}} \leq \int_{|t| \leq 2, \frac{1}{2}<s<2} \frac{d t d s}{s^{2}}=\mathscr{O}(1)
$$

for $\lambda \rightarrow 0^{+}$. Considering the contribution of each integral we have just calculated and observing that

$$
\lambda^{-4} \lesssim \lambda^{-\beta}
$$

for $0<\lambda \leq 1$, if $\beta \geq 4$ we conclude the proof.

\section{Acknowledgments}

I am very indebted to Professors Fabio Nicola and Elena Cordero for discussions and remarks which improved this paper in an essential way.

\section{References}

1. A. Cauli, F. Nicola, A. Tabacco Strichartz estimates for the metaplectic representation, arXiv:1706.03615, (2017).

2. E. Cordero, F. De Mari, K. Nowak, A. Tabacco, Analytic features of reproducing groups for the metaplectic representation, J. Fourier Anal. Appl. 12, no. 2, 157-180, (2006).

3. E. Cordero, F. De Mari, K. Nowak, A. Tabacco, Reproducing groups for the metaplectic representation, Pseudo-differential operators and related topics, 227-244, Oper. Theory Adv. Appl., 164, Birkhäuser, Basel, (2006).

4. E. Cordero, F. De Mari, K. Nowak, A. Tabacco, Dimensional upper bounds for admissible subgroups for the metaplectic representation, Math. Nachr. 283, no. 7, 982-993 (2010).

5. E. Cordero, A. Tabacco, Triangular subgroups of $S p(d, R)$ and reproducing formulae, J. Funct. Anal. 264, no. 9, 2034-2058, (2013).

6. E. Cordero, K. Gröchenig, F. Nicola, L. Rodino, Generalized metaplectic operators and the Schrödinger equation with a potential in the Sjöstrand class, J. Math. Phys. 55, no. 8, 081506 (2014).

7. E. Cordero, F. Nicola, Some new Strichartz estimates for the Schrödinger equation, J. Differential Equations 245, no. 7, 1945-1974, (2008).

8. E. Cordero, F. Nicola, Strichartz estimates in Wiener amalgam space and applications to the Schrödinger equation, Math. Nachr. 281, no. 1, 25-41, (2008).

9. E. Cordero, F. Nicola, Metaplectic representaiton on Wiener amalgam space and applications to the Schrödinger equation, J. Funct. Anal. 254, no.2, 506-534, (2008). 
10. M. Cowling, Sur les coefficients des représentations unitaires des groupes de Lie simples. Analyse harmonique sur les groupes de Lie (Nancy-Strasbourg, France, 1976-78), II, Lecture Notes in Math. 739, Springer, Berlin, 132-178, (1979).

11. M. Cowling, Herz's "principe de majoration" and the Kunze-Stein phenomenon. Harmonic analysis and number theory (Montreal, PQ, 1996), 73-88, CMS Conf. Proc., 21, Amer. Math. Soc., (1997).

12. M. Cowling, U. Haagerup, R. Howe, Almost $L^{2}$ matrix coefficient, J. Reine Angew. Math. 387, 97-110 (1988).

13. F. De Mari, K. Nowak Analysis of the affine transformations of the time-frequency plane, Bull. Austral. Math. Soc., Vol. 63, 195-218, (2001).

14. M. A. de Gosson, Symplectic Methods in Harmonic Analysis and in Mathematical Physics, Birkhäuser Basel, (2011).

15. M. A. de Gosson, The Wigner Transform, Advanced Textbooks in Mathematics, World Scientific, (2017).

16. K. Gröchenig, Foundations of Time-Frequency Analysis, Birkhäuser Basel, (2001).

17. T. Tao, Nonlinear dispersive equations: local and global analysis, American Mathematical Society, (2006). 